\title{
OBJECT-BASED VHSR IMAGE CLASSIFICATION USING MULTIBAND COMPACT TEXTURE UNIT DESCRIPTOR
}

\author{
K. Djerriri ${ }^{\mathrm{a},{ }^{*}}{ }^{*}$, A. Safia $^{\mathrm{b}}$, R. S. Cheriguene ${ }^{\mathrm{a}}$, H. S. Rahli ${ }^{\mathrm{a}}$, M. S. Karoui ${ }^{\mathrm{a}}$ \\ ${ }^{\text {a }}$ Centre des Techniques Spatiales, Département Observation de la Terre, Algeria; \\ ${ }^{\mathrm{b}}$ Centre for Research and Applications in Remote Sensing (CARTEL), Department of Geomatics, Sherbrooke University, Canada; \\ kdjerriri@cts.asal.dz
}

KEY WORDS: Multispectral images, Object-based Image Analysis, Multiband Compact Texture Unit

\begin{abstract}
:
In remote sensing, texture is commonly used to support spectral information particularly when spectral signatures of class of interest are similar. It is usually extracted using panchromatic band instead of multispectral bands. This is because panchromatic band has rich texture content due to its fine spatial resolution. Recent space-borne and pansharpening techniques can deliver multispectral images with a submetric resolution which are also good candidates for texture analysis. The difficulty in extracting texture in multispectral images is the fact that existing and widely used methods are limited to analyzing spatial relationship between pixels in a single band at a time. When multispectral images are used texture characterization is usually performed by analyzing spatial relationships in each spectral band independently. This ignores inter-band spatial relationships which can be a source of valuable source of information.

This paper evaluates the capability of a recently proposed method named multiband compact texture unit. This method extracts texture by characterizing simultaneously spatial relationship in the same band and across the different bands. This evaluation is performed in the context of object-based classification paradigm using WorldView-2 image of a forest area. For that image-objects were generated through superpixel segmentation. Classification in the object-feature space is performed suing $\mathrm{K}$ nearest neighbor algorithm. The proposed approach is compared to two groups of methods. The first group includes texture methods that use only spatial relationships in the same band: Gabor features wavelets and Granulometry. The second group includes methods that use intra-band and inter-band spatial relationships: integrative gray-level co-occurrence matrix, opponent Gabor features and opponent local binary patterns.

Experimental results show that texture extracted using both intra-band and inter-band spatial relationship improves the classification accuracy compared to when it is extracted in each spectral band independently. Among the methods of the second group that use both intra-band and inter-band spatial relationships, the multiband compact texture unit method produces the best results.
\end{abstract}

\section{INTRODUCTION}

Texture has been widely used for the analysis of remote sensing data particularly when spectral information cannot deliver sufficient results. It has been used to analyze medium spatial resolution images and it continues to play an increasing role in Very High Spatial Resolution (VHSR) images due to its capability to decrease the effect of high intra-class variability that characterizes VHSR images.

Most of the previous works on texture analysis have focused on extracting texture information using panchromatic bands due to their finer spatial resolution compared to multispectral ones. However, with the improvement of the spatial resolution of VHSR images, there is a growing interest on extracting texture from multispectral bands. Unfortunately, most of the existing texture methods were developed to extract texture from a single band and there is no general agreement on how to deal with images of many spectral bands. A widely adopted solution for extracting texture from multispectral images is to use existing monoband texture methods by analyzing each band independently. However, reducing the texture of multispectral images to a set of independent grayscale texture represents a very simplified solution as it does not take into account the multidimensional nature of image pixels.

Recently the authors in Safia and He (Safia and He, 2015a) introduced the concept of multiband texture as an extension of the concept of grayscale texture: "Multiband texture is defined as the spatial distribution of the tonal variations in the same band plus the spatial distribution of the tonal variations across different bands". The extraction of multiband texture by analyzing both intra- and inter-band spatial interactions simultaneously in many bands results in high calculation complexity. This is the main reason why the few available multiband texture methods are restricted to extracting intra- and inter-band spatial interactions between only two bands, which is an important limitation. Safia and He in (Safia and He, 2015a), proposed a new multiband texture descriptor which can extract multiband texture between a large numbers of bands simultaneously. The new descriptor is based on the Compact Texture Unit (C-TU) model proposed by the authors for monoband texture (Safia and He, 2013a) which has the advantage of having low computational complexity.

The process of texture extraction is time and resources consuming because it is performed locally using a moving window centered at each pixel in the image. The use of VHSR images for texture analysis makes the situation even harder. In recent years, object-based approach in contrast to pixel-based one has gained a great interest in the remote sensing community. When applying object-based method to image classification, images are decomposed to objects and after that classification will be conducted in object-feature space. Imageobjects as the basic classification unit were generated through image segmentation. Many studies reported that object-based approach can be an alternative to classic pixel-based image analysis particularly for VHSR images (Blaschke, 2010a).

This paper presents an evaluation of the multiband compact texture unit descriptor for simultaneous intra-band and interband texture analysis in an object-based classification scheme using WorldView-2 data in a forest environment. Segmentation step is performed using superpixels approach proposed in

* Corresponding author 
(Achanta, Shaji, Smith, Lucchi, Fua and Süsstrunk, 2012). To evaluate the pertinence of multiband texture features, the stateof-art texture features such as Gray-Level Co-Occurrence Matrix (GLCM), Gabor features and Local Binary Patterns (LBP) are also extracted for comparison purpose. Different feature descriptors were compared by means of classification accuracy. A simple K-nearest neighbors (KNN) classifier is employed for the classification in object-feature space.

\section{METHODS}

In this research, object-based image analysis is adopted. It is performed in three steps: superpixel based image segmentation, multiband compact texture unit feature extraction and supervised classification using KNN classifier.

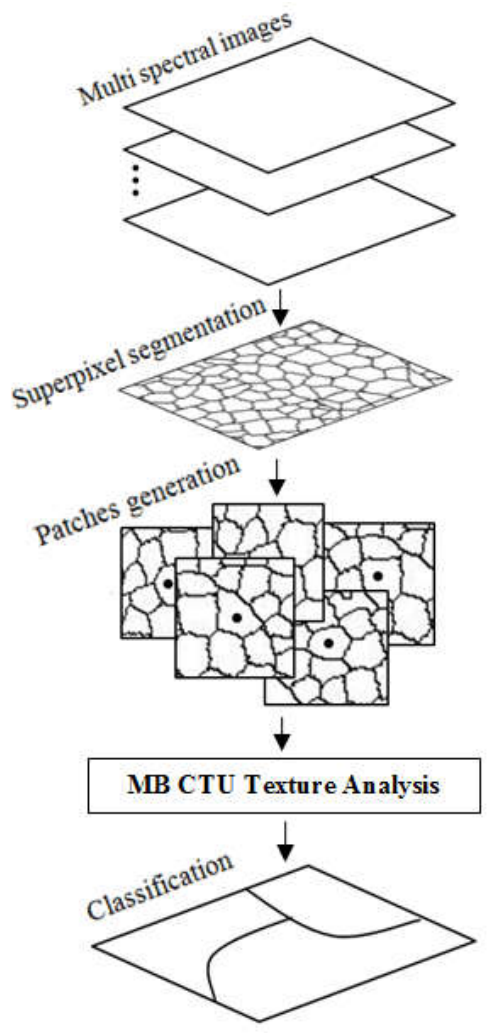

Figure1. Flowchart of the proposed methodology

\subsection{Image Segmentation}

Texture extraction is done generally over fixed size moving window. In this paper texture analysis is performed based on patches derived from superpixels (Achanta, Shaji, Smith, Lucchi, Fua and Süsstrunk, 2012). In order to take into account the limits between the different classes in the original image, we compute superpixels with the SLIC algorithm (Achanta, Shaji, Smith, Lucchi, Fua and Süsstrunk, 2012). Superpixels typically cover the whole image; they are distributed regularly with respect to the nature of the input image. The desirable variation of superpixels size is preferably small and the boundary of superpixels has to match the natural boundary of the different objects present in the image. The parameters of the superpixel extraction are tuned in order to get around 100 200 pixels by superpixel. The patches are 64 by 64 pixels subimages. One patch is generated for each superpixel. Figure1 presents the process of patch creation.

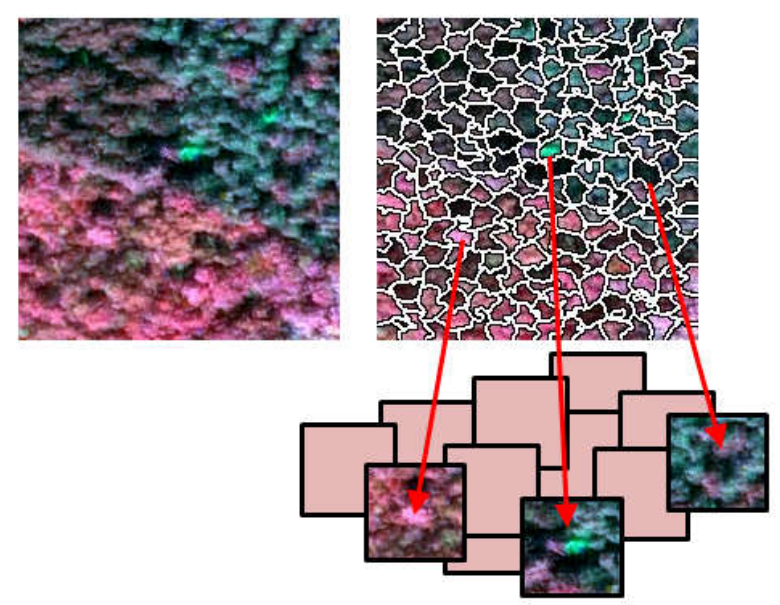

Figure2. Generation of patches from image

\subsection{Compact Texture Unit Analysis}

The compact texture unit was proposed to reduce the computational complexity of the "texture spectrum" texture analysis approach (Wang and He, 1990a), while maintaining equivalent performances. The image is scanned using a moving window.

\subsubsection{Monoband C-TU descriptor}

At each position, $\mathrm{N}$ (spatial resolution of the C-TU model) differences are calculated around the central pixel. The set $\Omega$ of all these differences over the entire image characterizes the local spectral variations contained in the image. The histogram giving the distribution of the elements in the set $\Omega$ is then divided into $\mathrm{M}$ regular subdivisions (spectral resolution of the C-TU model); each of them is assigned a unique code. Thus each local neighborhood is transformed into its equivalent spatial pattern called the Compact Texture Unit (C-TU).

A Compact Texture Unit Number (NC-TU) is assigned to each C-TU using the following sum formula:

$$
N_{C-T U}=\sum_{i=1}^{N} \zeta_{M, N}^{\Omega}\left(v_{i}-v_{0}\right)
$$

Where, $\mathrm{M}$ is the spectral resolution of the C-TU model, $\mathrm{N}$ : the spatial resolution of the C-TU model, $\mathrm{v}_{\mathrm{i}}$ : value of a given neighbourhood pixel, $\mathrm{v}_{0}$ : value of the central pixel, $\Omega$ is the set of the local spatial variation in the image and $\zeta_{\mathrm{MN}}^{\Omega}$ : function that associates $\mathrm{N}$ and $\mathrm{M}$ to a unique code.

The set of codes depends on the parameters $\mathrm{N}$ and $\mathrm{M}$. The authors in Safia and He (2013a) provided these codes for different spatial resolutions $\mathrm{N}$, and different spectral resolutions $\mathrm{M}$. The statistics of the occurrence frequency function of all the NCTU in the image or in a moving window provide a powerful tool for texture characterization. This is similar to the way with which an histogram allows spectral characterization. Figure 3 
shows the results of this transformation starting from a local neighborhood to the generation of the corresponding $\mathrm{N}_{\mathrm{CTU}}$.

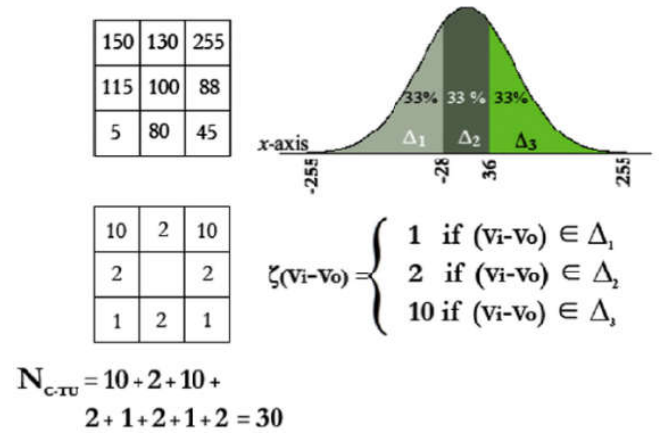

Figure 3. Transformation of a $3 \times 3$ neighborhood into a C-TU number using spectral resolution: $\mathrm{M}=3$ (Safia and $\mathrm{He}, 2013 \mathrm{a}$ ).

\subsubsection{Multiband C-TU descriptor}

The multiband descriptor extends the idea of monoband C-TU model to multispectral images. For that instead of using relative values of pixels in the same spectral band, it uses pixel values from all available spectral bands. To illustrate that, let's consider a 3 by 3 pixels local window in a three band image Figure 4(a). Spectral values in this window can be reorganized in order to form a new multiband neighborhood as follows. The central pixel of this multiband neighborhood can be taken as the value of the central pixel of the local window in the first spectral band. Twenty four neighbors can then be attributed to this new multiband neighborhood with their values taken as the values of the eight neighbors of the local window in the three spectral bands. This 24-pixel multiband neighborhood is shown in Figure 4(b).

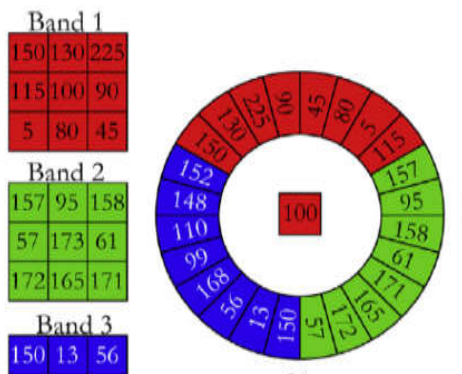

(b)

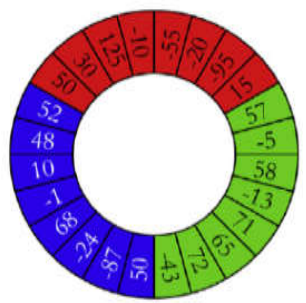

(c)
15811099
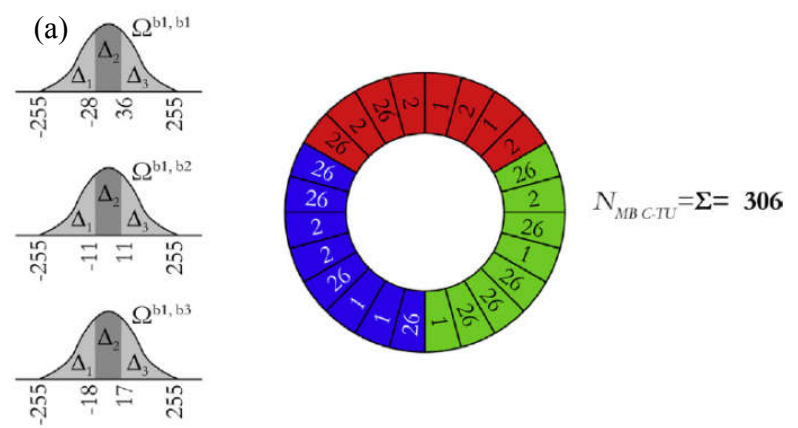

Figure 4. Extraction of the multiband C-TU descriptor for a three-band image using a $3 \times 3$ pixel multispectral neighborhood (Safia and $\mathrm{He}, 2015$ ).
The relative measures of pixel values in this 24-pixel multiband neighborhood, conducted exactly as in the monoband C-TU descriptor allows the characterization of different local spatial relation types. Indeed, when the central pixel from spectral band 1 is compared with the eight pixels from the same band, it is the classic intra-band local spatial relations which are characterized. This defines intra-band texture of spectral band 1 . When the central pixel is compared to the eight pixels from spectral band 2, it is the local inter-band spatial relations which are characterized. This defines inter-band texture of the spectral band 1 as viewed from the perspective of spectral band 2 . Similarly, comparison with pixels neighbors from band 3 leads to inter-band texture of spectral band 1 as viewed from the perspective of spectral band 3. In this way, a multiband neighborhood with pixels values from $p$ spectral bands contains $p$ types of local spectral variations: $p-1$ inter-band local variations plus one intra-band local variations. Result of the intra- and interband relative measures for the three-band case is presented in Figure 4(c).

\section{STUDY AREA AND USED DATA}

The validation of the proposed approach was conducted using pan-sharpened WorldView-2 multispectral image, acquired overthe Eastern Townships of Quebec, Canada, in August 2010. For that a subset of 1000 by 1000 pixels for which a detailed ground truth was available has been selected as test image. The subset represents typical forest landscapes with different pure and mixed forest stands (Figure 5).

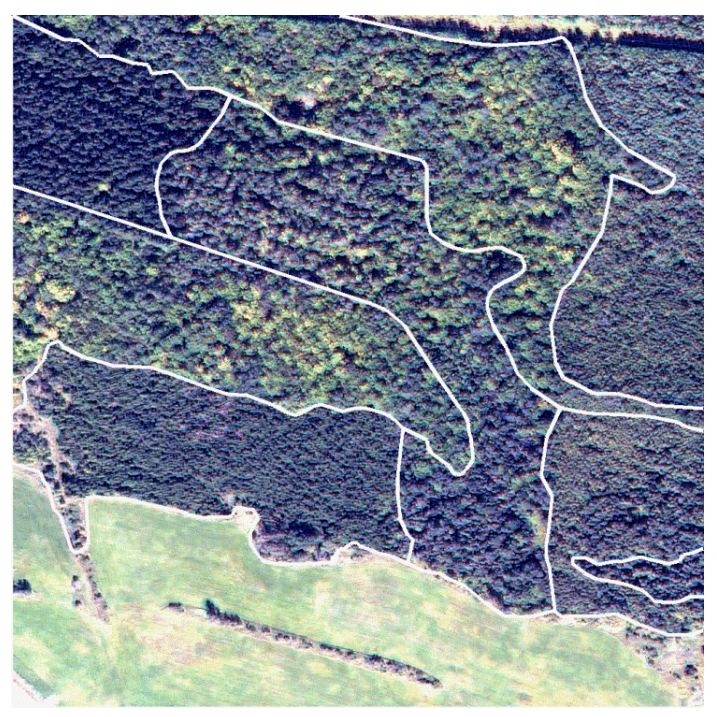

Figure 5. True color composite of the used image.

\section{EXPERIMENTS AND RESULTS}

After SLIC based segmentation, each superpixel was classified using a supervised nearest-neighbor rule (1-NN) with Euclidean distance algorithm (Altman, 1992). Training data were collected for each class in the test image. Continuous ground locations in test site showed important intra class texture variations. During the classification process, the p-bands compact textureunit numbers images were scanned with the generated superpixels. For each superpixel in the segmentation, the histogram of $\mathrm{N}_{\mathrm{MB}}$ C-TU was calculated for a patch of 64 by 64 pixels around its centroid and compared those of the training dataset. The pixels 
of the superpixels were labeled as the class that minimizes the Euclidean distance.

Classification accuracy assessment was conducted to assess the benefit compare of characterizing texture as a set of intra- and inter-band spatial interactions compared to the classic strategies that use only intra-band spatial interactions. To provide an idea about the performance of the multiband C-TU a comparison of existing methods that use inter-band and intra-band spatial relationships is presented. The overall correct classification and Kappa index provided a measure of agreement between the classifications produced and the ground data maps. These ground maps have the same size as the test images to allow a pixel by pixel accuracy assessment based on all pixels in the test images.
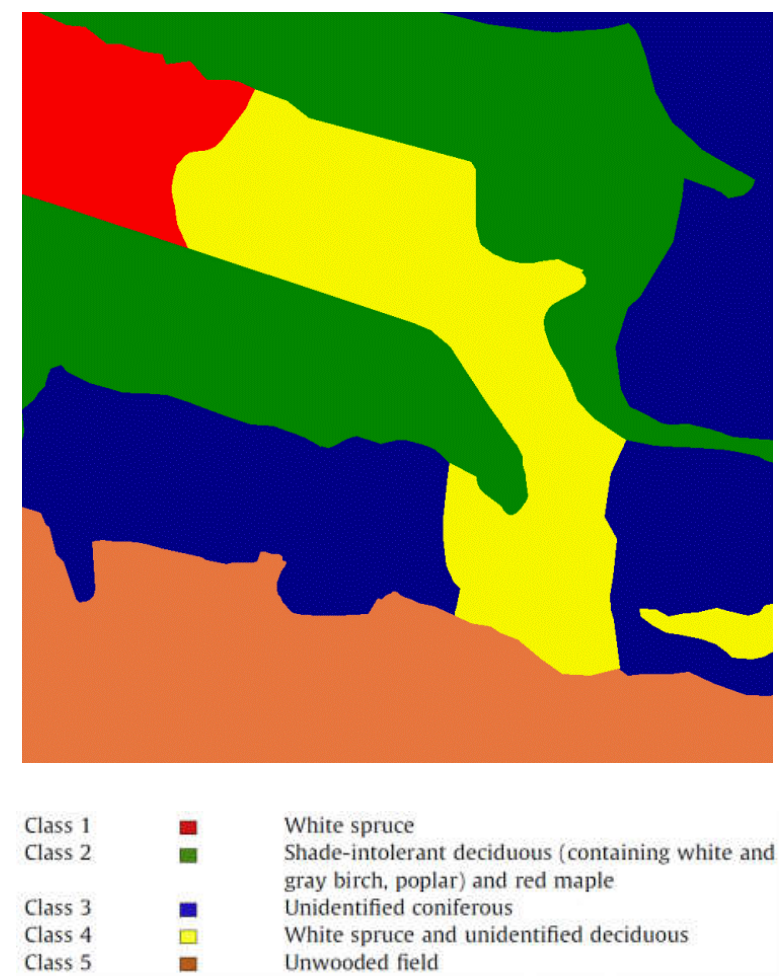

Figure 6 . Ground truth of the study area

The results of the object-based classification using multiband C-TU descriptor have been compared to six other texture features. Three of them use only intra-band characterization techniques: Gabor, wavelets and Granulometry features. The remaining three methods use intra-band and inter-band spatial relationships: integrative co-occurrence matrices, opponent Gabor features and opponent color local binary patterns (Bianconi, Harvey, Southam, Fernández, 2011). The comparison study was conducted using three bands image (nir, red and green spectral bands). Some of the resulting classified images are given in Figure. 7. The accuracy in terms of classification rate and Kappa index are summarized in Table 1.

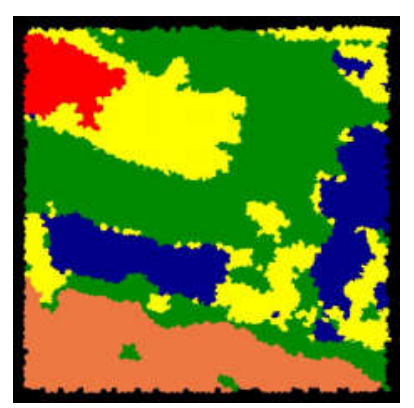

a. GLCM

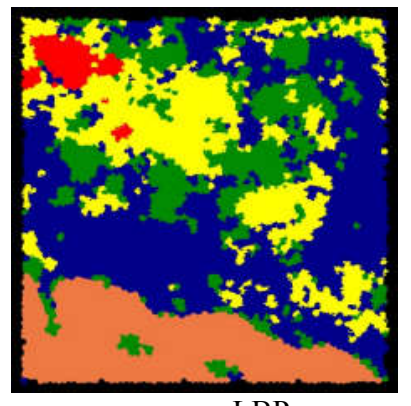

c. LBP

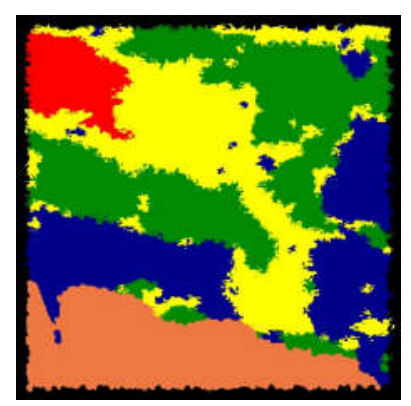

b. $\mathrm{MB} \mathrm{C}-\mathrm{TU}$

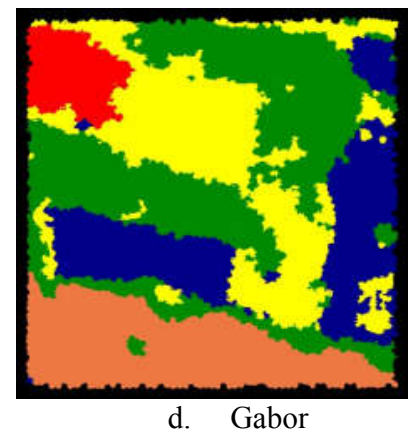

Figure 7 .Classifications of the used image

\begin{tabular}{|c|c|c|c|c|}
\cline { 2 - 5 } \multicolumn{1}{c|}{} & \multicolumn{4}{c|}{ Intra-bands } \\
\cline { 2 - 5 } \multicolumn{1}{c|}{} & Gabor & Wavelet & \multicolumn{2}{c|}{ Granulometry } \\
\hline OA (\%) & 72.83 & 66.94 & \multicolumn{2}{c|}{77.31} \\
\hline Kappa & 0.647 & 0.565 & \multicolumn{2}{c|}{0.704} \\
\hline & \multicolumn{4}{c|}{ Intra \& inter-bands } \\
\hline OA (\%) & 74.27 & $\mathbf{8 1 . 0 0}$ & 60.92 & 77.31 \\
\hline Kappa & 0.662 & $\mathbf{0 . 7 5 3}$ & 0.488 & 0.705 \\
\hline
\end{tabular}

Table 1.Classification rates of the conducted classifications

Table 1 shows that except for OLBP method, using both intraband and inter-band spatial relationships for texture analysis improves the classification rate compared to the classic intraband texture. For example the MB C-TU recorded an improvement of $14.06 \%$ compared the classic intra-band texture extracted using wavelet. The comparison between the three texture methods that use intra-band and inter-band spatial relationships shows that the multiband C-TU recorded the highest performances in terms of overall accuracy and kappa index. The improvement was $20.1 \%, 6.73 \%$ and $3.69 \%$ compared to the opponent LBP, IGLCM and opponent Gabor methods respectively. 


\section{CONCLUSION}

Texture of multispectral images is usually processed by analyzing each spectral band independently. In this work, an object-based classification has been conducted, where the basic processing units were patches of $64 \times 64$ pixels created around the segments from superpixel segmentation. The texture descriptor named multiband $\mathrm{C}-\mathrm{TU}$ was used to describe texture of multispectral images as multiband spatial information, which is the result of intra-band and inter-band spatial interactions. This descriptor is based on the idea of characterizing texture using relative pixel values in a multispectral neighborhood instead of a monoband neighborhood. The multibandC-TU descriptor can be extracted with low computationally complexity. The experimental results were promising and showed that extracting texture of multispectral images as a multiband texture is more efficient than the classic approaches based on only intra-band spatial interactions.

\section{REFERENCES}

Achanta, R., Shaji, A., Smith, K., Lucchi, A., Fua, P., \&Süsstrunk, S. (2012). "SLIC superpixels compared to state-ofthe-art superpixel methods". IEEE transactions on pattern analysis and machine intelligence, 34(11), 2274-2282.

Altman, N. S. (1992). "An introduction to kernel and nearestneighbor nonparametric regression". The American Statistician 46 (3): 175-185.

Bianconi, F., Harvey, R., Southam, P., \&Fernández, A. (2011). "Theoretical and experimental comparison of different approaches for color texture classification" .Journal of Electronic Imaging, 20(4), 043006-043006.

Blaschke, T. (2010). Object based image analysis for remote sensing. ISPRS journal of photogrammetry and remote sensing, 65(1), 2-16.

Safia, A., \& He, D. C. (2013). "Improving the Texture Spectrum Model Using a Compact Texture Unit Descriptor". Journal of Communication and Computer, 2, 234-251.

Safia, A., \& He, D. C. (2015). "Multiband compact texture unit descriptor for intra-band and inter-band texture analysis". ISPRS Journal of Photogrammetry and Remote Sensing, 105, 169-185.

Wang, L., \& He, D. C. (1990). "Texture classification using texture spectrum. Pattern Recognition”, 23(8), 905-910. 